\title{
El pensamiento de Maurice Halbwachs
}

\author{
Maurice Halbwachs's thought
}

Eguzki URTEAGA

Universidad del País Vasco

eguzki.urteaga@ehu.es

Recibido: 01-09-2010

Aceptado: 07-12-2010

\section{Resumen}

Gran figura de la sociología francesa de la primera mitad del siglo XX, Maurice Halbwachs ha dejado detrás de sí una obra de una gran riqueza, que va del estudio de la morfología social al de la psicología colectiva y que aborda temas tales como el consumo, las clases sociales, la memoria de los grupos y de las sociedades, el suicidio, la vida urbana, la religión o la demografía. Consta también de numerosos escritos metodológicos dedicados a la cuantificación en ciencias sociales y revela al público francés unos autores extranjeros esenciales, a imagen de Werner Sombart, Thorstein Veblen, Max Weber, Robert Park, Ernest Burgess o John M. Keynes. Este artículo presenta varios conceptos fundamentales de la obra de Maurice Halbwachs entre los cuales figuran los de morfología social, clase social y conciencia de clase.

Palabras clave: Halbwachs, sociología, memoria colectiva, suicidio, clase obrera.

\section{Abstract}

Great figure of the French sociology of the first half in the 20th century, Maurice Halbwachs has realized a work of a great richness, which goes from the 
study of the social morphology to that of the collective psychology and which analyse matters such as consumption, social classes, memory of the social groups, suicide, urban life, religion or demography. It consists also in numerous methodological writings dedicated to the quantification in social sciences and reveals to the French public a few foreign essential authors, like Werner Sombart, Thorstein Veblen, Max Weber, Robert Park, Ernest Burgess or John M. Keynes. This article presents several fundamental concepts of Maurice Halbwachs's work between which appear those of social morphology, social class and class conscience.

Key words: Halbwachs, sociology, collective memory, suicide, working class.

\section{Introducción}

¿Cómo dar cuenta de la complejidad del pensamiento de Maurice Halbwachs? Alexandre considera que la formación de la conciencia social constituye la problemática central de la sociología del autor francés. En numerosas circunstancias, debido a su formación filosófica, ha utilizado ese concepto, bien aplicándolo a las clases sociales, bien explicando las interacciones entre memoria individual y memoria colectiva, bien mostrando cómo los datos de la morfología social influyen en los comportamientos. Por su parte, Namer (2000) considera que "el objeto total finalizado de la vida intelectual" de Halbwachs es la memoria social. Los primeros rasgos son perceptibles en los artículos dedicados a Bernstein (1905) y en ciertos pasajes de La clase obrera (1912), antes de que acceda a ese estatus en Los marcos sociales de la memoria (1925) y en La memoria colectiva (1950).

Semejantes puntos de vista, que consisten en privilegiar la génesis de las ideas, en situarse ampliamente en una perspectiva histórica y en hacer resaltar la coherencia interna del corpus, no carecen de interés. Presentan sin embargo el inconveniente de ocultar la riqueza y la originalidad de la obra de Halbwachs: la prodigiosa e incesante curiosidad intelectual que expresa, la exigencia empírica y metodológica que aplica a cada observación, las pasarelas establecidas entre las diferentes ciencias sociales. Omiten también recordar otro concepto fundamental en el pensamiento de Halbwachs, el de morfología social, a pesar de ser omnipresente en sus trabajos desde su tesis doctoral sobre las expropiaciones. Por lo tanto, es indispensable abordarlo así como los conceptos de clase social y de conciencia de clase. Las dificultades planteadas por la noción de memoria social/memoria colectiva son analizadas y profundizadas por Namer (1987 y 2000). 


\section{La morfología social}

$\mathrm{Si}$, a lo largo de sus investigaciones, Halbwachs no ha cesado de hacer referencia a la morfología social, ha conseguido aclarar ese concepto, que fue utilizado tanto por Émile Durkheim como por Marcel Mauss, en la obra que tiene ese mismo título (1938). En La división del trabajo social (1893), Durkheim veía en las transformaciones del orden material de la sociedad (incremento de la población, multiplicación de las ciudades, extensión de las posibilidades de comunicación) la causa profunda de esta división: la densidad creciente de los grupos sociales, aproximando a los individuos, permitiéndoles especializarse en una actividad y haciéndolos más complementarios, contribuye a la profundización del proceso de división del trabajo. En Las reglas del método sociológico (1895), indicaba que la extensión y la composición de las sociedades, el número de las partes que las constituyen y el nivel de integración suscitan, especialmente en la organización política, unas instituciones particulares.

Ha definido con la mayor precisión lo que entiende por morfología social en el segundo tomo de la revista L'Année sociologique (1899). Es el substrato sobre el cual se fundamenta la vida social, determinada tanto en su grandeza como en su forma por la masa de individuos que componen la sociedad, la manera según la cual están dispuestos sobre el suelo, la naturaleza y la configuración de las cosas de todo tipo que afectan las relaciones colectivas. Según Durkheim, la morfología social, que convierte en una especialidad de la sociología, no debe ser solamente una simple ciencia de la observación puesto que debe ser explicativa. El substrato social no es una totalidad formada y dada y corresponde al sociólogo, reuniendo las aportaciones de la historia, de la geografía y de la demografía, comprender cómo se ha constituido. ¿En función de qué evolucionan los territorios nacionales? ¿Por qué las fronteras de los Estados toman tal o tal forma? ¿Por qué las sociedades prefieren unas situaciones centrales o periféricas? ¿Cuáles son los hechos que dan lugar a las ciudades y a los pueblos? Como "la constitución de ese substrato afecta, directamente o indirectamente, a todos los fenómenos sociales", la explicación de los hechos sociales debe fundamentarse ampliamente en esa "ciencia de las formas materiales de la sociedad" que es la morfología social.

Escribiendo la Morfología social (1938), el proyecto de Halbwachs consiste no solamente en retomar, enriqueciéndolo y completándolo, ese principio de explicación de lo social sino también en ampliar su perspectiva y alcance explicativo. Para ello, empieza introduciendo una distinción entre "morfología social restringida", la de los fenómenos de población, y "morfología en sentido amplio", contemplada no tanto a escala de la sociedad en su conjunto sino a nivel de las comunidades, que corresponden a otras tantas sociologías particulares (familiar, religiosa, política o económica). 
La morfología en el sentido amplio hace referencia a tres morfologías particulares. Por ejemplo, en la morfología religiosa, las grandes agrupaciones de población son indispensables para comprender los grandes fervores. Si las grandes ciudades pueden considerarse como globalmente desfavorables a la práctica de las religiones, ya que históricamente han sido sus focos iniciales y por las facilidades de contacto que han supuesto, han acelerado su difusión. En cuanto a la morfología política, se basa en el recordatorio del rol de las comunicaciones marítimas en la aparición de las democracias y el de las ciudades en la elaboración de las constituciones y de las organizaciones locales. Por último, la morfología económica hace intervenir las clases sociales. Tienen cierta tendencia a separarse en el espacio y la historia de las ciudades está hecha de arreglos sociales sucesivos.

El estudio de la morfología social restringida es la oportunidad para Halbwachs de ofrecer un condensado de los conocimientos demográficos de la época. La presentación sobre las "condiciones espaciales", la población considerada en sus relaciones con el suelo, y el "movimiento natural de la población, todo lo que se refiere a las variables que ordenan los nacimientos y los fallecimientos, moviliza las contribuciones de los demógrafos tanto antiguos (John Graunt, Thomas-Robert Malthus) como modernos (Gini, Lotka, Kuczynski).

La gran novedad con respecto al estado anterior de la reflexión sobre el concepto de morfología social estriba en un enfoque renovado de la manera según la cual es susceptible de actuar sobre la sociedad. Para Halbwachs, esa influencia no es, como lo sugiere Durkheim, directa y mecánica, sino que transita por la conciencia social: "Comprendamos bien, ahora, que las formas materiales de la sociedad actúan sobre él, no tanto en virtud de una presión física o de un cuerpo que actuaría sobre otro cuerpo, sino por la conciencia que tenemos como miembros de un grupo que perciben su volumen, su estructura física, sus movimientos en el espacio. Hay un cierto nivel de pensamiento o de percepción colectiva que podría denominarse un dato inmediato de la conciencia social"l.

La vida de un grupo social está hecha de representaciones colectivas que se constituyen por la concienciación de su estructura y de sus movimientos. Desde las primeras páginas del libro, Halbwachs, cita el ejemplo de la familia. El sexo, la consanguinidad y la aproximación en el espacio de ciertos individuos no son suficientes para constituir una familia. Ésta solo existe a través de las relaciones de parentesco, las relaciones afectivas, la cohesión entre las generaciones, las tradiciones y el espíritu que la estructuran y unen a sus miembros. Todos estos elementos atestiguan de la presencia de una conciencia colectiva. Sucede lo mismo para el conjunto del cuerpo social. "Tanto la morfología social como la sociología, se refieren ante todo a las representaciones colectivas. Si fijamos nuestra atención sobre estas for-

${ }^{1}$ Halbwachs, M. (1938), Morfología social. Paris: Armand Colin, p.182. 
mas materiales, es con el fin de descubrir, detrás de ellas, toda una parte de la psicología colectiva. Porque la sociedad se inserta en el mundo material, y el pensamiento del grupo encuentra, en las representaciones que le provienen de estas condiciones espaciales, un principio de regularidad y de estabilidad, así como el pensamiento individual necesita percibir el cuerpo y el espacio para mantenerse en equilibrio"2. En ese sentido, Halbwachs propone un análisis mucho más matizado y menos determinista que Durkheim tomando en consideración las representaciones colectivas.

\section{Las clases sociales}

El gran mérito de Halbwachs, a pesar de las críticas que ha recibido, consiste en haber conseguido hacer entrar las clases sociales en la sociología durkheimiana. En La clase obrera (1912), presenta su propio trabajo como un intento de superación de la oposición, según él ficticia, entre las concepciones del economista Gustav Schmoller (1838-1917), que define las clases sociales por la profesión y los puestos ocupados por los individuos en la sociedad, y la del economista y sociólogo Karl Bücher (1847-1930), para el cual son identificables a través de las diferencias de renta y de patrimonio. Más allá del proyecto inicial, es ante todo por comparación con unos enfoques más clásicos del tema, las de Karl Marx y de Max Weber, que la originalidad del análisis de Halbwachs resalta de manera nítida.

Entre los historiadores de la sociología, la puesta de manifiesto de las oposiciones entre las concepciones de Marx y de Weber se ha convertido en un ejercicio clásico. Recordemos brevemente sus aspectos fundamentales.

Los escritos de Marx, y especialmente El manifiesto del partido comunista (1848), La lucha de clases en Francia (1850), El 18 Brumario de Luis Napoleón Bonaparte (1852) y diferentes pasajes del Capital (1867), contienen unas divergencias en cuanto al número y a la composición de las clases sociales pero se refieren a una concepción realista y subjetiva. Es realista en el sentido de que la existencia de las clases sociales, directamente observable, resulta del modo de producción capitalista. Éste, en el marco de la relación productiva que instaura, reparte unas posiciones de clase. El antagonismo fundamental se sitúa entre los explotadores, propietarios de los medios de producción, capaces de imponer una relación salarial de explotación y de retener un excedente sobre el valor creado por el trabajo (la plusvalía), y los explotados, que solo disponen de su fuerza de trabajo. Como realidad objetiva y observable, la clase existiría "en sín".

Además, Marx escribe que no podría existir ninguna clase social sin una conciencia de pertenencia a esta por parte de sus miembros. Solo se constituye defini-

2 Halbwachs, M. (1938), Morfología social. Paris: Armand Colin, p.12-13. 
tivamente en clase por una concienciación de su existencia, una afirmación consciente de solidaridad y un programa preciso de acción histórica. Ello significa que a través de los conflictos que lo oponen a la burguesía, la clase obrera consigue comprender la lógica de explotación que se le impone y concienciarse de sus propios intereses. Puede organizarse y preparar el derrocamiento del capitalismo y existe entonces, de manera subjetiva, una clase "por sí".

En la sociología de Weber ${ }^{3}$, las clases sociales, que se distinguen de los grupos con estatus y de aquellos que se fundamentan en el prestigio, solo son una de las modalidades, que se refieren al orden económico, de las estratificaciones sociales que se encuentran en las sociedades humanas. La identificación de las clases sociales supone por parte del observador una operación de división de la sociedad en distintos grupos, realizada no directamente en razón de un principio de realidad, sino por la elección de criterios lógicos de diferenciación aplicables a cualquier sociedad humana. Esta concepción nominalista se opone a la de Marx.

Más precisamente, Weber considera que una clase social puede definirse por la presencia de recursos económicos similares y de intereses comunes entre unos individuos. Entonces, es posible reconocerles una "situación de clase" (a través de las relaciones que mantienen con otras clases en función de su patrimonio, de su renta y de su acceso a la cultura) y una "condición de clase" (la probabilidad que unos intereses similares se encuentren). Está claro que Weber no asigna a las clases sociales, como lo hace Marx, un rol histórico. En el mejor de los casos, puede ser autor de acciones colectivas.

En la medida en que todos los análisis de Halbwachs sobre las clases sociales se fundamentan en unos resultados de encuestas donde las categorías utilizadas en estas últimas son retomados como tales, no se encuentra en su obra ningún equivalente a la perspectiva weberiana que consiste en concebir una división en clases que se fundamenta en tipos ideales. Desde ese punto de vista, los trabajos de Halbwachs se aproximan a los de Marx. Este último es citado regularmente en su asignatura sobre las clases sociales, mientras que Weber lo cita solo una vez y a propósito de su distinción entre las formas de poder. A primera vista, ciertas similitudes, tendientes al carácter "realista" del enfoque de las clases y a la referencia permanente a la conciencia de clase, se imponen. Friesdmann (1946) ha observado que interesándose por la sociedad industrial, las relaciones de producción que influyen sobre la mentalidad, la sensibilidad y las conductas sociales de los individuos, Halbwachs "se aproxima a la sociología marxista".

Movilizando a numerosos resultados de encuestas realizadas con los obreros, analizando de cerca sus presupuestos y el reparto de su gasto corriente, visitando las viviendas si fuera necesario, Halbwachs se ha dotado de los medios necesarios para

3 Weber, M. (1922), Économie et société. Paris: 10/18. 
poder hablar de realidades objetivas y concede una gran trascendencia a las descripciones relativas a los niveles de vida, a las condiciones de trabajo en las fábricas y a las manifestaciones de representaciones colectivas (consumo, frecuentación, formas de sociabilidad).

La segunda similitud con Marx, perceptible en las primeras páginas de La clase obrera (1912), es la referencia a la conciencia de clase como condición sine qua non de su existencia. Halbwachs plantea como axioma que una clase social no existe mientras no percibe el lugar que le está asignado en la jerarquía social. "Nos parece contradictorio suponer que una clase existe sin concienciarse de ella misma. Nada impide a un historiador o a un sociólogo distinguir en una sociedad varios grupos teniendo en cuenta unas similitudes y unas diferencias exteriores o aparentes entre sus miembros. Pero, semejantes clasificaciones tienen tendencia a ser lo más a menudo artificiales si no nos preocupamos ante todo de la actitud y de las disposiciones colectivas de las personas. Llamar clase a un conjunto de individuos en el cual una conciencia de clase no se ha desarrollado y no se ha manifestado, es no designar ningún objeto social"4.

Estas aparentes similitudes no impiden a Halbwachs proponer un análisis de las clases sociales que difiere profundamente, de manera reivindicada, de la de Marx. Dos diferencias fundamentales los separan. La primera es la relación establecida entre la pertenencia de clase y el modo de consumo y el nivel de socialización y no, como lo hace Marx, el lugar ocupado en las relaciones de producción. Mientras que la sociología marxista está dominada por la presencia de un antagonismo fundamental entre las clases que constituyen la sociedad, Halbwachs privilegia la dimensión de la integración en el seno de cada grupo social. Muestra lo que reúne a sus miembros, cuáles son las condiciones materiales de vida compartidas, los comportamientos y los sentimientos comunes, las solidaridades entabladas. Relega a un segundo plano no solamente la cuestión del contacto entre las clases sino también su carácter conflictivo.

La segunda se refiere a la cuestión del número de clases sociales. Durante mucho tiempo, esa cuestión no parece haber preocupado a Halbwachs. Hay que esperar Las clases sociales (1937) y El esbozo (1955) para que la estratificación social sea contemplada en su conjunto, sin que se justifique verdaderamente el modo de división elegido.

Las clases sociales se distinguen unas de otras, no tanto por su posición en el proceso de producción como por su nivel de vida en el sentido planteado por Halbwachs, es decir por su manera de consumir, sus gustos, sus preferencias y su nivel de participación en la vida social, en sus manifestaciones más elevadas e intensas. En función de las sociedades, puede tratarse, bien del acceso a unos bienes simbólicos e inmateriales, los misterios, los ritos sagrados, bien del acceso al

${ }^{4}$ Halbwachs, M. (1912), La classe ouvrière et les niveaux de vie. Paris: Félix Alcan, p.11. 
poder y a las responsabilidades, bien del acceso a la riqueza y a los bienes. En todos los casos, el acceso a los bienes, materiales o no, determina una jerarquía social. Efectivamente, entendido de manera tan amplia, el "nivel de vida" reviste una doble dimensión. No significa solamente una satisfacción más o menos grande de las necesidades, sino también, a través del acceso a los bienes, un nivel de integración social. Como lo escribe Halbwachs: "es siempre con respecto a los bienes considerados como más importantes en cada tipo de sociedad que se definen las clases" 5 .

En la perspectiva de una comparación con la teoría marxista, esta definición de las clases sociales, aunque ponga de manifiesto el consumo, no elimina por ello cualquier referencia a las condiciones de producción. Conviene no olvidar que el proyecto inicial de Halbwachs, en su deseo de desmarcarse de las tesis de Schmoller y Bücher, consistía en establecer una relación entre trabajo y consumo. "Supongamos que bajo la influencia de la vida social las necesidades naturales $\mathrm{u}$ orgánicas hayan evolucionado, se hayan convertido en artificiales y más complejas y también en menos imperiosas. Habitualmente sustraídas a la acción de la sociedad, obligados a un gasto notable de fuerzas físicas, a una actividad sobre todo fisiológica, los obreros sienten quizás con más intensidad esas necesidades naturales, en su forma básica: pueden convertirse para ellos en necesidades esenciales. Por otra parte, numerosas necesidades sobre todo sociales solo se desarrollan gracias a una educación de una larga duración: el contacto permanente con la sociedad, la inteligencia en sus formas más elevadas, el sentido y el gusto de las ventajas que procuran a los que conocen el éxito"6.

Semejantes indicaciones incitan a releer las páginas dedicadas a las características comunes del trabajo obrero. Evocan el carácter astringente y fundamentalmente deshumanizante del trabajo industrial en el inicio del siglo XX. "Sean cual sean los materiales, el obrero es el que, en y por su trabajo, sigue estando aislado ante la naturaleza, se enfrenta a las fuerzas inanimadas: el obrero es todavía la persona cuya actividad debe plegarse al ritmo de las máquinas cuyos gestos deben vincularse y conformarse a los de sus compañeros de trabajo, de manera a reproducir el juego mecánico de las máquinas, como si solo se pudiese domar la materia sometiéndola a unas leyes tan regulares e impersonales que las leyes naturales. Por último, el obrero es el que, peón u obrero, debe abstenerse de cualquier iniciativa y aspirar solamente a convertirse o seguir siendo un instrumento seguro y bien adaptado a una tarea simple o compleja pero siempre monótona" 7 .

Contrariamente a lo que se podría pensar, semejantes notaciones no conducen ni a la elaboración de una teoría de la alimentación, ni a una condena de la explotación capitalista. Halbwachs evita cuidadosamente cualquier utilización del térmi-

\footnotetext{
5 Halbwachs, M. (1912), La classe ouvrière et les niveaux de vie. Paris: Félix Alcan, p. III.

6 Halbwachs, M. (1912), La classe ouvrière et les niveaux de vie. Paris: Félix Alcan, p. 132.

${ }^{7}$ Halbwachs, M. (1912), La classe ouvrière et les niveaux de vie. Paris: Félix Alcan, p.118-119.
} 
no de alienación. Cuando se trata de evocar las relaciones de producción, utiliza expresiones neutrales: los obreros están predispuestos al trabajo de la materia, la sociedad delega parte de sus miembros a la realización de tareas materiales.

Resulta de todo ello una concepción no conflictiva de las relaciones de clase. Constituye una segunda diferencia fundamental con respecto a la concepción marxista. Se trata de una visión original del funcionamiento de la sociedad que se fundamenta no tanto en un esquema de oposición de clase sino de distanciamiento y de alejamiento social, ya que la sociedad es comparada a un pueblo reunido en torno a un "núcleo central". Las condiciones de trabajo impuestas a los obreros influyen en su manera de consumir pero es solamente en el contacto con la sociedad exterior, cuando están sumergidos en la verdadera vida social, que realizan la inferioridad de su misión. "Los obreros se convierten en unos instrumentos que, como todos los que utiliza el ser humano, tienden a identificarse cada vez más a las fuerzas materiales y mecánicas de la naturaleza. Contraen, por lo tanto, unas maneras de pensar y unas costumbres opuestas a las maneras de pensar y a los hábitos sociales. Asimismo, no es en esta zona de la actividad industrial, sino en la sociedad propiamente dicha, donde las personas, consumiendo, satisfacen igualmente sus necesidades. Sea cual sea su origen y su naturaleza, las distinciones de clase se manifiestan y adquieren un sentido social"8.

Según Halbwachs, la conciencia de clase no se forma en la fábrica, en la esfera del trabajo, sino en su exterior, a través del contacto con la sociedad, y se revela por la mediación del consumo. "Encargado" o "delegado" a las tareas materiales, aislado de los demás trabajadores durante su ejecución, el obrero se encuentra desvinculado de la sociedad. La conciencia de clase emerge, por lo tanto, fuera de la fábrica, lo que provoca un desplazamiento que se acompaña de un postulado de no-conflictividad, del centro del análisis social. "No se debe buscar el verdadero principio de separación de las clases en el lugar de trabajo. El paso alternativo de la zona del consumo al de la producción y viceversa despierta y alimenta en ellos, cuando trabajan, la sensación de una inferioridad social. Solo puede comprenderse cómo nace su conciencia de clase limitándose al término trabajo. Es porque los que trabajan han consumido y consumirán, antes de ser sacados de la sociedad, que perciben el carácter anómalo de su situación. Comparan la vida social de afuera, tejido más o menos denso de relaciones humanas, con el horizonte material de sus vidas en la fábrica, con el trabajador industrial"9.

Para justificar su propósito, Halbwachs utiliza un argumento discutible pero que le parece suficiente: los obreros, separados por las máquinas y concentrados en la ejecución de su tarea, no se enfrentan entre ellos ni con los empresarios porque las necesidades de la producción los aíslan en el espacio y los separan materialmente.

\footnotetext{
8 Halbwachs, M. (1912), La classe ouvrière et les niveaux de vie. Paris: Félix Alcan, p.118-119.

${ }^{9}$ Halbwachs, M. (1912), La classe ouvrière et les niveaux de vie. Paris: Félix Alcan, p.128-129.
} 
Para volver a las relaciones entre las clases sociales y retomar la metáfora que Halbwachs utiliza en las primeras páginas del libro, las clases se distinguen unas de otras manteniéndose a una distancia variable de un "hogar central" correspondiente a "la vida social más intensa que se pueda representar", es decir a las actividades valorizadas y consideradas como nobles ${ }^{10}$. "Se observa, a medida que uno se eleva a una u otra clase, que los grupos están cada vez más integrados, es decir que sus miembros se encuentran cada vez más insertados en una red de relaciones sociales, religiosas, políticas, económicas, etc., en función del tipo de sociedad. Se puede interpretar de dos maneras: bien se dirá que la sociedad en su conjunto tiende a superarse, que la vida social inicialmente difusa, dispersa, sometida a la acción de muchas fuerzas dispersas, se concentra poco a poco, se recoge en torno a un foco que ha encendido él mismo y que alimenta; bien se considerará que la sociedad hace un esfuerzo penoso y constante para distenderse, como si tuviese que desvincular de ella muchas fuerzas de opresión que la encierran y la ahogan y que sus partes más vecinas se encuentran cada vez más alejadas del núcleo central".

Ese alejamiento relativo determina la jerarquía entre las clases sociales. Cada una puede definir, a través de una participación desigual a la vida social, por la distancia que la separa del "núcleo central". Las más favorecidas, las más instruidas y las más refinadas, en las cuales las relaciones interpersonales son más ricas, son las que más se aproximan. Los obreros, cortados del resto de la sociedad durante el tiempo de trabajo y reducidos a una sociabilidad familiar y de proximidad (la calle, el barrio) el resto del tiempo, son mantenidos al margen. En esas condiciones, la visión de una sociedad dominada por unos conflictos de clase encuentra difícilmente su lugar. Los obreros se definen más como unos primitivos que como unos dominados y unos explotados susceptibles de oponerse a unas clases dominantes. Más tarde, Halbwachs ha utilizado en varias ocasiones la metáfora del "núcleo central".

Para concluir, utilizando una terminología que no es desconocida para Halbwachs a propósito de la estratificación social, propone un esquema de oposición de tipo centro-periferia. De modo que nos encontremos ante el primer enfoque de la exclusión social. "El conjunto de los habitantes de la ciudades que se distinguen claramente de todos los demás en la medida en que están vinculados y menos adaptados que ellos a la sociedad" 11 . Conviene subrayar la actualidad de dicha frase, más aún sabiendo que se refiere a los urbanos. Escribiéndola, Halbwachs solo intentaba definir el grupo de los obreros, pero podría aplicarse sin dificultad a las poblaciones marginadas y precarizadas de los suburbios urbanos o "barrios sensibles". Abre un debate complejo entre inserción e integración.

10 Baudelot, C., Establet, R. (1994), Maurice Halbwachs. Consommation et société. Paris: PUF, pp.3645.

${ }^{11}$ Halbwachs, M. (1912), La classe ouvrière et les niveaux de vie. Paris: Félix Alcan, p.135. 
En ese sentido, el análisis de las clases sociales en Halbwachs se distingue de todas las demás, que ponen de manifiesto el carácter productivista y tecnicista de las sociedades occidentales. Realiza una inversión, al beneficio del segundo término, de la relación entre el ámbito de la producción y el del consumo. Describiendo las posiciones respectivas de las clases sociales bajo la forma de conjuntos más o menos alejados de un "núcleo central", simbolizando las manifestaciones más intensas y más prestigiosas de la vida social, Halbwachs sustituye a una visión piramidal y vertical de la sociedad, una visión horizontal. Abre la vía a un análisis en términos de exclusión. Por esas razones, ofrece un modelo alternativo a las presentaciones habituales de las clases sociales.

Aunque se trate de enfoques muy diferentes en sus intenciones y en su contenido, se distingue tanto del análisis marxista (oposición entre clase dominante y clase dominada) como de la herencia de la sociología americana de las comunidades de la primera mitad del siglo XX, la que ha establecido, tras las investigaciones de Llyod Warner, la distinción entre clases altas (upper class), medias (middle class) y bajas (lower class). La comparación con la sociología de las comunidades es interesante a ese nivel. Como la de Halbwachs, ha intentado caracterizar las clases y sus actividades basándose en la relación entre el ser humano y la materia. En sus famosas encuestas sobre la ciudad media en Estados Unidas, Robert y Helen Lynd (Middletown, a Study in Contemporary American Culture, 1929; Middletown in transition, 1937) han repartido los habitantes en dos grupos: la "clase obrera", reúne a los que se ganan la vida trabajando con unos objetos, utilizando unos instrumentos materiales para fabricar unos bienes, y la "clase de empresarios", constituida por las personas cuyas funciones, a través de la promoción y de la venta de bienes, de servicios y de ideas, consiste en entrar en relación con otras personas.

Sin embargo, la contribución de Halbwachs a la teoría de las clases sociales ha generado ciertas críticas. Así, es a través de un artificio, invocando la distancia física y moral que separa los que cumplen las diferentes funciones en las empresas, que aparta el estudio de los conflictos sociales susceptibles de estallar en el mundo laboral. No se trata de un reproche que se puede dirigir exclusivamente al sociólogo francés, ya que pone de manifiesto una de las debilidades de la sociología durkheimiana. Atenta a las normas y a los valores sociales, describe los fenómenos de integración social, tiende a infravalorar o a ignorar las situaciones de dominación, de poder y de conflicto. Preocupado en cernir la normalidad, Durkheim se había interesado sobre todo a los elementos de inercia y de regularidad de la vida social. Insistía en la constancia de las relaciones que se establecen entre los hechos sociales. La manera según la cual enuncia las leyes del suicidio constituye uno de los mejores ejemplos.

Uno de los méritos de Halbwachs ha sido introducir un cierto número de elementos novedosos en esta sociología, a imagen del estudio de las clases sociales, 
pero ello ha supuesto caer en ciertas simplificaciones. La teoría del "núcleo central", que reduce las relaciones de clase a una cuestión de distancia social, es una de ellas, así como la supuesta y postulada unidad de la clase obrera. "Sin duda no hay ninguna clase más homogénea, precisamente porque la vida social es más reducida, menos complicada, y también porque el intervalo que la separa de los demás grupos, desde ese punto de vista, está muy marcado, que la clase obrera"12.

Semejante afirmación significa, ni más ni menos, negar la existencia de subgrupos y de subculturas en el seno de la clase obrera. No en vano, su diversidad, bajo la perspectiva del oficio, de la calificación, de la contratación (obreros de origen rural que practican a menudo la doble actividad y obreros urbanos) o de organizaciones socio-profesionales (sociedades de socorro mutuo, sindicatos), es bien conocida. Numerosos analistas han hecho resaltar la espontaneidad, la vitalidad y el dinamismo de la que hacen gala las clases obreras ${ }^{13}$. Esa impresión está confirmada por el hecho de que la clase obrera está considerada como carente de memoria. Halbwachs elude igualmente la cuestión, sin embargo fundamental, de la formación de la clase obrera. Mencionando la organización industrial del trabajo, sitúa su estudio a un cierto nivel de desarrollo del capitalismo pero no llega a preguntarse desde hace cuánto tiempo los obreros presentan las características subrayadas ni cómo han aparecido y si estas son o no inamovibles.

Estos errores de valoración se deben al hecho de que la sociología de Halbwachs, retomando la formula de Verret (1972), "se para a las puertas de la fábrica". Preocupado sobre todo por el hecho de ver en el trabajo industrial una de las modalidades, extrema, de la relación que se establece entre el hombre y la materia, Halbwachs se aleja de una verdadera sociología del trabajo atenta, no solamente a las modalidades del trabajo industrial, sino también a las relaciones sociales y a las solidaridades que se establecen en el seno de la fábrica, a las organizaciones y a los movimientos que se constituyen en esa ocasión, a las ideologías que circulan. Es solamente en la segunda mitad de los años 1930 que Halbwachs empieza a hablar de sindicalismo.

Visiblemente, Halbwachs ha reconocido ciertas realidades. Según sus descripciones, los obreros se pliegan perfectamente a las condiciones de trabajo que les son prescritas a pesar de que se siga observando en los talleres, como consecuencia de las resistencias y de los márgenes de autonomía individual que subsisten, un diferencia entre los métodos elegidos por la dirección y la realidad del trabajo realizado. Las relaciones industriales y el universo concreto del trabajo constituyen otro ámbito de la sociedad en el que el sociólogo no se aventura. El reconocimiento de

\footnotetext{
12 Halbwachs, M. (1912), La classe ouvrière et les niveaux de vie. Paris: Félix Alcan, p. XIII.

13 Hoggart, R. (1970), La culture du pauvre. Etude sur le style de vie des classes populaires en Angleterre. Paris: Minuit.
} 
ese distanciamiento deliberado en muy explícito en Los marcos sociales de la memoria. "La puerta de la fábrica representa exactamente para el obrero la línea de separación entre las dos partes de su vida diaria. Si sigue estando entreabierta, es sobre todo después de la jornada de trabajo: una parte de las costumbres de pensar o de no pensar, que conlleva el contacto exclusivo con la materia, refluye en la zona de la sociedad donde vive el obrero cuando está fuera del taller. Cuando vuelve a su lugar de trabajo, siente que deja detrás de sí un mundo para entrar en otro, y que no hay ninguna comunicación entre ambos".

La teoría de las necesidades obreras presentada por Halbwachs, confirmada por las estadísticas, plantea asimismo un problema de interpretación. El sociólogo francés asimila las decisiones tomadas por el consumidor, bajo la influencia de su participación en la producción material, a una posición de retirada hacia la vida social y de rechazo de alinearse sobre las prácticas de otros entornos sociales. ¿Hasta qué punto, lo que es presentado por Halbwachs como unas elecciones que se fundamentan en unas representaciones sociales de clase, una manera de contemplar y de jerarquizar las necesidades, no es el resultado de las coacciones objetivas padecidas por los consumidores como consecuencia de la escasez de ciertos bienes? ¿La debilidad relativa del gasto de vivienda no sería la consecuencia de la rareza y de la carestía de la vivienda en los entornos urbanos o la constatación de las penurias encontradas en el mercado inmobiliario? Curiosamente, ese problema que era mencionado al final de Expropiaciones (1909), a través de la evocación de las operaciones de los especuladores (más dispuestos a construir unas viviendas burguesas que unas viviendas obreras), desaparece en los libros posteriores dedicados a las necesidades obreras.

Percibiendo así la vida de los obreros, Halbwachs cede a su etnocentrismo de clase. Así como Durkheim veía en las religiones primitivas las formas básicas de las religiones de todas las sociedades, Halbwachs se representa al obrero como a un primitivo en el seno de las sociedades industriales. Más que en los desarrollos dedicados a la burguesía, es en los pasajes que tratan de la nobleza y de sus tradiciones que el autor desvela lo que constituye finalmente, según él, el grado más elevado de la vida social al que concede tanto valor: el hecho de poder escapar a las coacciones técnicas vinculadas a una actividad profesional y de poder dedicarse a las recepciones, a las mundanidades, a las delicias de la existencia desvinculada de ciertas obligaciones materiales. En ciertos entornos, como la antigua nobleza y ciertas fracciones de la burguesía, sobre todo cuando la función ejercida permite unos contactos con el prójimo, la vida social se impone fácilmente a las obligaciones profesionales y para-profesionales. En otros entornos, tales como las clases media y obrera, los individuos tienen más dificultades para desvincularse. "En los entornos familiares y mundanos efectivamente las preocupaciones generales, las que son comunes al mayor número de personas, se imponen a las demás: es entonces cuando lo social 
se crea bajo sus formas más puras. Desde ahí circula en dirección de otros grupos"14.

Escribiendo esas frases, Halbwachs se opone a la Teoría de la clase del ocio (1899) desarrollada por Thorstein Veblen que denunciaba el ocio como una actividad improductiva y el consumo ostentoso de una "clase ociosa" presentada como desvinculada de las obligaciones industriales y que se considera como superior. Una concepción tan elitista de las relaciones sociales es sorprendente bajo la pluma del sociólogo galo, militante socialista y saludado por su simplicidad, su humildad y su sentido de la compasión. En realidad, la personalidad de Halbwachs es más compleja de lo que parece. Se aclara parcialmente a la lectura de los detalles que cuenta sobre su campaña de candidatura al Collège de France. Descubrimos un personaje bien introducido, que se desenvuelve con soltura en el mundo intelectual y político parisino, que moviliza los recursos de una amplia red de conocimientos y de amistades establecidas durante su paso por la Escuela normal superior, los gabinetes ministeriales y las misiones oficiales. Hijo de un profesor agregado, aunque no originario de esa clase, Halbwachs se ha visiblemente forjado una idea aristocrática de la estratificación social. Finalmente, por una inversión completa de situación, esta parte de la obra del discípulo de Durkheim se convierte en una demostración de la dificultad a tratar los hechos sociales como cosas.

A pesar de esas críticas, el análisis de las clases sociales realizada por Halbwachs es valiosa. Desde hace ciertos años, la subida del desempleo masivo, el incremento de las desigualdades sociales, la vulnerabilidad creciente de la posición de las clases medias en la sociedad francesa, ha favorecido una renovación del interés por los análisis en términos de clases sociales ${ }^{15}$. Esa tendencia lleva lógicamente consigo mismo una necesidad de renovación de la teoría de las clases sociales. En un momento en el cual la reflexión teórica se halla solicitada, la lectura de un autor como Halbwachs resulta ser estimulante. La literatura sociológica contemporánea muestra que no se trata únicamente de un deseo. Es el caso, por ejemplo, de Alain Touraine en su análisis de la exclusión contemporánea y de sus manifestaciones urbanas donde evoca "el paso de una sociedad vertical, que denominados la sociedad de clases, con unas personas arriba y otras a bajo, a una sociedad horizontal donde lo importante es saber si nos encontramos en el centro o en la periferia. (...) Lo que se denomina simbólicamente, el suburbio, es precisamente esa zona de gran incertidumbre y de tensiones, en la cual las personas no saben si caerán del lado del in o del lado del out"16.

\footnotetext{
14 Halbwachs, M. (1912), La classe ouvrière et les niveaux de vie. Paris: Félix Alcan, p. 332.

15 Chauvel, L. (2001), « Le retour des classes sociales ? ", La revue de l'OFCE, n79.

Bouffartigue, P. (2004), Le retour des classes sociales. Paris: La Dispute.

Lemel, Y. (2004), Les classes sociales. Paris: PUF.

16 Touraine, A. (1991) ; «Face à l'exclusion », in Citoyenneté et urbanité. Paris: Esprit.
} 
Con la referencia a los suburbios urbanos, Touraine introduce una dimensión espacial que está ausente en Halbwachs, por lo menos en sus escritos sobre las clases sociales. Pero, la sustitución de una representación vertical de la sociedad por una representación horizontal recuerda el esquema de los círculos concéntricos dispuestos alrededor del "núcleo central" imaginado por este último. El paralelismo puede ser prolongado. Hace algunas décadas, el "final de las clases sociales" había sido decretado a la vista de las consecuencias de la crisis económica contemporánea. Ha hecho perder cierta pertinencia a las referencias antiguas basadas en el trabajo, la relación salarial, la explotación, las formaciones históricas de la militancia sindical y política y las ideologías que las acompañaban. Los nuevos conflictos (los problemas de los suburbios urbanos, las violencias urbanas, las dificultades de convivencia en los barrios sensibles) hacen pensar en la aparición de nuevas formas de enfrentamiento que se sustituyen al antagonismo histórico entre burguesía y proletariado. Los conflictos de origen interétnico, racial y religioso agravados por la segregación social urbana, se habrían sustituido a los que se producían en el mundo laboral. Por lo tanto, la exclusión habría puesto punto final a la lucha de clases.

En todo caso, conviene admitir que la lectura de los textos de Halbwachs, que definen las clases sociales de otra forma que por su participación en la producción y las sitúa unas con respecto a otras desvinculándose de los esquemas habituales, abre unas interesantes pistas de reflexión.

\section{La conciencia de clase}

Se trata de uno de los conceptos más problemáticos de la sociología marxista. Supuestamente, debe permitir el paso entre filosofía y discurso de la acción revolucionaria. La referencia a la conciencia de clase da lugar en Marx a unas indicaciones por lo menos imprecisas y contradictorias. En La introducción de la filosofía del derecho de Hegel (1844), escribe que conviene "formar" el proletariado. En El manifiesto del partido comunista (1848), sugiere "constituirlo en clase" con el fin de que tome conciencia de la necesidad de una revolución radical y de su rol histórico. Las realidades diarias de la vida obrera, cuyas coacciones son descritas por Marx, no parecen ser suficientes para hacer emerger espontáneamente entre los obreros una conciencia de clase cuya existencia sigue siendo de hecho virtual. Designar a una clase como tal, dirigirse a ella y pretender dirigirla, es admitir que ya estaría constituida por una conciencia de clase preexistente. Esta concepción plantea no solamente un problema lógico sino también político. En la medida en que el proyecto de acción asignado a la clase obrera, el cuestionamiento y el derrocamiento del capitalismo, se sitúa a largo plazo, lo que plantea la cuestión del arbitraje con unos intereses a más corto plazo. Acaba siendo difícil saber lo que debe 
ser la motivación de la acción obrera inmediata y quién debe decidir. Esta concepción plantea igualmente un problema filosófico que estriba en el conflicto entre la filosofía materialista, que sirve de fundamento al marxismo, y una filosofía idealista, que supone recurrir a una elevación de la conciencia de clase.

En los dos artículos que ha dedicado a Edouard Bernstein (1850-1932), pensador social-demócrata alemán conocido por su crítica del marxismo ortodoxo, Halbwachs (1905) saluda la pertinencia de una perspectiva que presenta para él el triple mérito de fundamentarse en la experiencia, de proceder de un simple pensamiento inductivo y de tomar en consideración "la experiencia concreta" de los obreros modernos. En esa óptica, rechaza reducir la conciencia de clase a una peculiaridad individual y psicológica identificada de manera más o menos intuitiva, lo que no es sorprendente bajo la pluma de un durkheimiano ortodoxo que era todavía en esa época. Rechaza igualmente conformarse con un pensamiento abstracto y deductivo que pretende tratar objetivamente y de manera definitiva la situación de clase. A partir de ese momento, Halbwachs dibuja los contornos de un programa cuya realización llevará varias décadas: vincular el estudio de la conciencia de clase a la noción de representación colectiva contemplada de manera inductiva a través de una acumulación de indicadores y de datos estadísticos.

Halbwachs siente la necesidad de recordar regularmente esta exigencia, como en ese pasaje del curso impartido sobre las clases sociales. "Para ciertos sociólogos, las opiniones y las representaciones colectivas son unas realidades del mismo modo que las cosas materiales. Por lo tanto, conviene ser prudente a la hora de fijar nuestra atención exclusivamente en las relaciones técnicas de producción, olvidando que hay algo todavía más importante y que no debe ser descuidado. Son las opiniones de la sociedad sobre la técnica y sobre otra cosa también. Es la propia sociedad entendida como un conjunto de representaciones"17. Esta perspectiva en el estudio de la conciencia de clase puede ser denominada de "experimental". Constituye la trama de La clase obrera (1912). Reduciéndola a una manifestación objetiva y medible, Halbwachs intenta superar una de las dificultades fundamentales de la teoría de las clases sociales.

Si Halbwachs considera que la conciencia de clase puede ser reducida a una dimensión económica, ésta es exterior a la producción. "En realidad, no es en la fábrica y durante las horas dedicadas al trabajo, que tanto el empresario como el obrero toman sobre todo conciencia de pertenecer a dos clases"18. Se revela por la mediación de los sentimientos en materia de satisfacción de las necesidades y de las valoraciones que le están vinculados. Como consecuencia de su aislamiento ante la materia durante su actividad profesional y de sus relaciones con los demás obreros

\footnotetext{
17 Halbwachs, M. (1937), Histoire de l'économie sociale. Les classes sociales. Paris: Centre de documentation universitaire, p.137.

18 Halbwachs, M. (1912), La classe ouvrière et les niveaux de vie. Paris: Félix Alcan, p.126.
} 
fuera de la fábrica, el obrero se aleja de la búsqueda de ciertas satisfacciones más activamente buscadas por otros grupos sociales y restringe el ámbito de sus necesidades. "Tomar conciencia de sí mismo, para una clase, es reconocer a qué nivel social se sitúa y es, posteriormente, presentarse con respecto a qué privilegios, a qué derechos, a qué ventajas, se miden estos niveles y se determina esta jerarquía. Cualquier representación de clase implica una doble valoración: la valoración del bien o de los bienes más importantes y los más apreciados en la sociedad considerada; la valoración del grado hasta el cual está permitido a los miembros de la clase satisfacer las necesidades que le están vinculadas"19. La clase obrera se conciencia, a la vez, del sistema jerárquico que estructura la sociedad en su conjunto y del lugar que ocupa en el seno de esa jerarquía.

Halbwachs intenta, por lo tanto, reduciendo su expresión a unos modos de consumo diferenciados, dar a esta conciencia de clase un contenido concreto, susceptible de ser analizado sociológicamente y objetivamente. La acumulación de estadísticas sobre los presupuestos y su análisis minucioso, que ocupan tantas páginas de La clase obrera, de La evolución de las necesidades y de un cierto número de artículos, no tienen otra finalidad que de revelar los sistemas de preferencias y los significados asociados al consumo, pruebas intangibles de la existencia de una conciencia común a los obreros. "Como consumidores, los individuos se procuran diversos bienes y satisfacen sus necesidades básicas. Pero las necesidades pueden ser consideradas como aún más sociales cuando su satisfacción se acompaña de un mayor número de pensamientos y de sentimientos colectivos. Si hay clases, conviene esperar que, en cada una de ellas, las diversas necesidades no están ni tan plenamente satisfechas, ni jerarquizadas de la misma manera: una parte fundamental del estudio de estos grupos consiste en determinar los "niveles de vida" clasificados según la satisfacción y el desarrollo desigual de las necesidades sociales y no sociales" 20 .

De esa manera, la conciencia de clase no se halla contemplada desde fuera, con una búsqueda de rasgos característicos elegidos de manera más o menos arbitraria por un observador exterior, sino a través de las representaciones sociales y de sus manifestaciones concretas. Por la mediación del reparto de sus necesidades, es posible ver cómo, a través de los sentimientos comunes que las animan, las personas se clasifican ellos mismos y se conciencian de las realidades de clase y de su lugar en la escala social. Pero, incluso aceptando ese análisis y reduciendo la conciencia de clase a esa dimensión económica objetiva, Halbwachs está lejos de haber resuelto todos los problemas planteados por la utilización de ese concepto. Considerándolo como un estado, lo aborda de manera totalmente estática, lo que plantea la cuestión de la formación. Analizada así, a través de las representaciones vinculadas al con-

19 Halbwachs, M. (1912), La classe ouvrière et les niveaux de vie. Paris: Félix Alcan, p.II.

${ }^{20}$ Halbwachs, M. (1912), La classe ouvrière et les niveaux de vie. Paris: Félix Alcan, p.IX. 
sumo, ¿la conciencia de clase está determinada por la renta? ¿Es por la mediación de los fenómenos de imitación entre miembros de una misma clase? ¿Por el hecho de que los contactos con los miembros de otras clases se encuentran limitados o inexistentes? ¿Estas representaciones son susceptibles de modificarse?

Las únicas respuestas aseguradas que Halbwachs aporta a esas cuestiones se refieren al primer punto, es decir al rol de la renta. En La clase obrera (1912) y posteriormente en La evolución de las necesidades (1933), muestra que los obreros y los empleados no reparten de la misma manera sus gastos, incluso cuando disponen de un presupuesto similar. Utilizando indistintamente los términos de necesidad y de consumo, Halbwachs realiza una confusión entre la causa y el efecto del fenómeno estudiado y se cierra cualquier posibilidad de preguntarse sobre el origen y la difusión de las necesidades. Es solamente en el último capítulo de su segunda obra que figuran ciertas indicaciones sobre la manera según la cual son susceptibles de formarse y de transformarse las costumbres de consumo.

Después de haber recordado una vez más que, incluso situado en un contexto de evolución de los presupuestos al consumo, la clase obrera no aumenta significativamente la parte del gasto de alojamiento. Halbwachs da las siguientes indicaciones. "En nuestras grandes urbes modernas, las personas de todas las clases se abastecen y compran en las mismos grandes tiendas, mientras que hay un mercado de las pertenencias burguesas y un mercado distinto de las viviendas obreras. Mientras que los mismos productos alimenticios están expuestos a la vista de todos, en los muestrarios, y que, en las calles, obreros y empleados se codean, se observan y por consiguiente se imitan en la manera de vestirse, los obreros no conocen las viviendas de los empleados y las comparaciones a este propósito solo pueden establecerse en el seno de cada clase" 21 .

Estas anotaciones identifican toda una serie de factores (la urbanización, la organización del comercio y de la distribución, el funcionamiento de los mercados, los fenómenos de clientela, de asistencia y de imitación) que estaban ausentes hasta entonces. No en vano, son fundamentales para la comprensión del consumo. La sociología del consumo ha podido averiguar posteriormente la pertinencia de esas hipótesis. Es lamentable que Halbwachs no se haya comprometido más aún es esta vía de investigación. De nuevo, la percepción de la realidad de las relaciones entre las clases sociales aparece como un punto débil de su análisis. En nombre de un argumento "morfológico" discutible, la segregación social y espacial urbana que separaba irremediablemente a los individuos que pertenecen a unos entornos sociales diferentes, postula una fuerte impermeabilidad de las clases sociales unas hacia otras.

${ }^{21}$ Halbwachs, M. (1912), La classe ouvrière et les niveaux de vie. Paris: Félix Alcan, p.145-146. 


\section{Conclusión}

La importancia de la obra de Halbwachs resulta, en primer lugar, del lugar que ocupa en la historia de la sociología francesa de la primera mitad del siglo XX. Al lado de Marcel Mauss y de François Simiand, Halbwachs aparece como un autor de primera importancia. Además de asumir la herencia intelectual de Emile Durkheim, ha sabido desmarcarse de ella, especialmente estableciendo unos vínculos entre psicología y sociología, proponiendo un enfoque más dinámico y más completo de los hechos sociales, introduciendo nuevas problemáticas y haciendo regularmente un uso perfectamente controlado y razonado del instrumento estadístico.

A este propósito, la referencia a un "segundo durkheimismo" encarnado por Halbwachs puede resultar equivocado. La expresión es excesiva a la vista de las diferencias existentes. Estas son suficientes para que la sociología de Halbwachs aparezca como otra cosa que una mera prolongación de Durkheim. Por el contrario, es demasiado débil para adherirse a la idea según la cual se habría producido un alejamiento pronunciado de los objetos de estudio privilegiados por la Escuela durkheimiana. En realidad, Halbwachs no ha cesado de hablar, incluso si ha contribuido a hacer evolucionar el concepto, de morfología social. En las clases que ha impartido al final de los años 1930 y en el inicio de los años 1940, ha recordado regularmente las reglas metodológicas durkheimianas. En cuanto a los Anales sociológicos, ha relanzado la revista de inspiración durkheimiana y ha retomado las secciones (sociología general, sociología religiosa, sociología jurídica y moral, etc.), tal y como habían sido fijados unos cuarenta años antes por Durkheim.

La obra de Halbwachs es igualmente importante por su contenido, especialmente por la fecundidad de las ideas y de las hipótesis que se expresan en ella, por la inmensidad del trabajo de apertura de nuevos campos para la sociología y el rigor científico sobre el cual se fundamenta. Merece no solamente ser redescubierta sino también enseñada y utilizada, ya que constituye una "sociología de nuestro tiempo"22. Halbwachs ha identificado unos temas que han sido retomados, a veces varias décadas después. El "método de los presupuestos" introducido por Halbwachs, tras los primeros intentos de Le Play y sus discípulos, ha sido utilizado de manera sistemática por el INSEE a partir de los años 1950. Antes que todo el mundo, fue un teórico de la exclusión y casi lo ha sido de la noción de habitus. Podríamos igualmente citar un esbozo de sociología de la elección del nombre en Los marcos sociales de la memoria, en el capítulo dedicado a la familia. Haciendo aparecer detrás del desorden aparente de la teoría social unas regularidades, relativas al consumo, a la nupcialidad, a los nacimientos, a los suicidios y a otros fenómenos, Halbwachs se ha aventurado, antes que los demás, en la teoría de la com-

22 Baudelot, C., Establet, R. (1994), Maurice Halbwachs. Consommation et société. Paris: PUF, p.121. 
plejidad. Los estudios sobre la memoria, que conocen un nuevo auge desde unos veinte años, se han convertido en la oportunidad de recordar otro aspecto, el más conocido actualmente de ese precursor.

Sus escasas biografías subrayan el hecho de que Halbwachs solo ha dirigido dos tesis doctorales. No habría tenido verdaderos discípulos, lo que explicaría el olvido del que ha sido víctima durante un largo periodo. Si ese hecho es indiscutible, está ampliamente compensado por los testimonios de los que, una vez convertidos en eminentes especialistas en sus disciplinas respectivas, han reconocido su deuda hacia él: Paul-Henry Chombart de Lauwe (sociología urbana), Georges Friedmann (sociología del trabajo), Marcel Roncayolo (geografías e historias urbanas), Alfred Sauvy (demografía y economía) o Jean Stoetzel (psicología social). Más allá de sus innumerables contribuciones a la sociología, Halbwachs ha establecido un diálogo y una colaboración con las demás ciencias sociales. Quizás haya venido la hora, haciendo jugar una de las leyes de la memoria colectiva que ha puesto de manifiesto, de reconocer el interés y valor de esta obra 23 .

\section{Bibliografía}

Alexandre, J. (1940-1948), « Maurice Halbwachs », Année sociologique, pp.3-10. Амiот, M. (1991), "Le système de pensée de Maurice Halbwachs », Revue de Synthèse, n¹12-2, pp.13-33.

Baudelot, C., Establet, R. (1994), Maurice Halbwachs. Consommation et société. Paris: PUF.

Bouffartigue, P. (2004), Le retour des classes sociales. Paris: La Dispute.

Bourdieu, P. (1987), "L'assassinat de Maurice Halbwachs », Visages résistance, $\mathrm{n}^{\circ} 16, \quad$ pp. 164-170, http://www.college-de-france.fr/media/ins_dis/ UPL41150_halbwbourd.pdf

Chauvel, L. (2001), « Le retour des classes sociales?», La revue de l'OFCE, nº 79 .

\footnotetext{
23 Cabe señalar que el escritor Jorge Semprun conoció personalmente a Maurice Halbwachs que fue su profesor en la Universidad de la Sorbona en París. En una lectura de su texto titulado Mal et modernité: le travail de l'histoire el 19 de junio de 1990, en el marco de las Conferencias Marc Bloch, Jorge Semprum habla de su relación con Maurice Halbwachs durante su deportación en el campo de concentración de Buchenwald. Tras el fusilamiento del historiador Marc Bloch por los nazis en SaintDenis-de-Formans, cerca de Lyon, el 16 de junio de 1944, Maurice Halbwachs le hablo ampliamente de él y del libro Les rois thaumaturges. Durante el otoño de 1944, se reunió cada domingo con Maurice Halbwachs. Se desplazaba al bloque $\mathrm{n}^{\circ} 56$, donde se encontraban los discapacitados y deportados inaptos para el trabajo. Allí se hallaban Maurice Halbwachs y Henri Maspero con los cuales mantenía unas intensas discusiones. Gravemente enfermo, Maurice Halbwachs apenas conseguía bajar las escaleras del bloque en el que se encontraba y, a veces, ni siquiera podía hablar. Agonizó en los brazos de sus compañeros hasta su muerte el 16 de marzo de 1945.
} 
Gresle, F. (1989), « Conscience sociale, représentations et mémoire collective chez Maurice Halbwachs », in Mentalités et représentations politiques. Aspects de la recherche. Roubaix: Edires.

HALBWACHS, M. (1905), « Remarques sur la position de problème sociologique des classes », Revue de métaphysique et de morale, $\mathrm{n}^{\circ} 13$, pp.890-905.

Halbwachs, M. (1908), « Budgets des familles », Revue de Paris, pp.534-562.

HaLBWACHS, M. (1912), La classe ouvrière et les niveaux de vie. Recherches sur la hiérarchie des besoins dans les sociétés industrielles contemporaines. Paris: Félix Alcan.

HaLBWACHS, M. (1914), « Budgets de familles ouvrières et paysannes en France en 1907 », Bulletin de la statistique générale de la France, octobre, pp.47-83.

Halbwachs, M. (1933), L'évolution des besoins dans les classes ouvrières. Paris: Félix Alcan.

HaLbWachs, M. (1937), Histoire de l'économie sociale. Les classes sociales. Paris: Centre de documentation universitaire.

Halbwachs, M. (1938), Morfología social. Paris: Armand Colin.

HaLBWACHS, M. (1939), "Les caractéristiques des classes moyennes », in Inventaires III. Les classes moyennes. Paris: Félix Alcan.

Halbwachs, M. (1939), "Conscience individuelle et esprit collectif », The American Journal of Sociology, n 44 , pp.812-822.

Halbwachs, M. (1955), Esquisse d'une psychologie des classes sociales. Paris: Marcel Rivière.

Hoggart, R. (1970), La culture du pauvre. Etude sur le style de vie des classes populaires en Angleterre. Paris: Minuit.

LeMel, Y. (2004), Les classes sociales. Paris: PUF.

LENOIR, R. (2001), «From Maurice Halbwachs to Pierre Bourdieu: social nature or naturalized social construction », International Journal of Contemporary Sociology, vol.38, $\mathrm{n}^{\circ} 1, \mathrm{pp} .41-53$.

LENOIR, R. (2003), "M. Halbwachs, Soziologie und demographie", in ST. EGGER (Hg), Maurice Halbwachs Aspekte des Werks, UVK Verlagsgesellschaft Mbh, Konstanz, pp. 115-143

MARCEL, J-C. (2002), " Maurice Halbwachs et les classes sociales », Alternatives économiques, $\mathrm{n}^{\circ} 208$, pp.77-81.

MARCEL, J-C. (2004) « Mauss et Halbwachs: vers la fondation d'une psychologie collective (1920-1945) », Sociologie et sociétés, vol. 36, n², pp.73-90. Montréal: Les Presses de l'Université de Montréal.

Martinez, E. (2008), "Bibliografía selecta de y sobre Maurice Halbwachs », Revista Anthropos: Huellas del conocimiento, $\mathrm{n}^{\circ} 218$, pp.50-54.

MontLiBERT DE, C. (1997), Maurice Halbwachs (1877-1945). Strasbourg: Presses Universitaires de Strasbourg. 
Montlibert DE, C. (2008), « Maurice Halbwachs à Strasbourg: une philosophie rationaliste en action », Revue des Sciences Sociales, n40, pp.82-93.

Mucchielli, L. (1999), «Un fondement du lien social: lé mémoire collective chez Maurice Halbwachs », Technologies, idéologies, pratiques. Revue d'Anthropologie des Connaissances, n²4, pp. 63-88.

Pequignot, B. (dir.) (2007), Maurice Halbwachs: le temps, la mémoire et l'émotion, Paris: L'Harmattan.

Topalov, C. (1997), « Maurice Halbwachs et les villes (1908-1912). Une enquête d'histoire sociale des sciences sociales ", Annales, Histoire et sciences sociales, vol. 53, n5, pp.1057-1083.

Touraine, A. (1991) ; «Face à l'exclusion », in Citoyenneté et urbanité. Paris: Esprit.

Verret, M. (1972), « Halbwachs ou le deuxième âge du durkheimisme », Cahiers internationaux de sociologie, n53, pp.311-336.

WEBER, M. (1922), Économie et société. Paris: 10/18.

Eguzki Urteaga

Departamento de Sociología 1

Universidad del País Vasco

eguzki.urteaga@ehu.es 